\title{
Obtención de recubrimientos de hidroxiapatita sobre titanio mediante el método biomimético ${ }^{(\cdot)}$
}

\author{
A. Paz*, Y. Martín*, L.M. Pazos**, M.B. Parodi**, G.O. Ybarra** y J.E. González*
}

\begin{abstract}
Resumen En el presente trabajo se realizó un estudio de la deposición de hidroxiapatita sobre un sustrato de titanio mediante el método biomimético. Para ello se empleó una disolución con elevado contenido de calcio y fósforo (SCS). Además, se realizó la activación del titanio a partir de la aplicación de procesos de oxidación con peróxido de hidrógeno y ácido clorhídrico con un posterior tratamiento térmico. La caracterización de los materiales empleados, así como de los recubrimientos obtenidos, se realizó mediante Espectroscopia Infrarroja (FT-IR), Difracción de Rayos X (DRX), Microscopia Electrónica de Barrido (MEB) y Espectroscopia de Energía Dispersiva de Rayos X (EDX). Como resultado del proceso de activación se formó un óxido de titanio hidratado. Sobre la superficie activada se logró obtener un recubrimiento de hidroxiapatita en un período de $24 \mathrm{~h}$, caracterizado por su elevada densidad y cristalinidad y por presentar un espesor entre $2-4 \mu \mathrm{m}$.
\end{abstract}

\section{Obtaining hydroxyapatite coatings on titanium by the biomimetic method}

\begin{abstract}
In this work, a study about the deposition of hydroxyapatite on a titanium substrate employing the biomimetic method is presented. A solution with high content of calcium and phosphorus (SCS) was used. In addition, activation of titanium with hydrogen peroxide and hydrochloric acid and a subsequent heat treatment was performed. The characterization of materials used and the coating obtained was carried out by Infrared Spectroscopy (FT-IR), X-ray Diffraction (XRD), Scanning Electron Microscopy (SEM) and Energy Dispersive X-ray Spectroscopy (EDX). As a result of the activation processes a hydrated titanium oxide was formed. On the active surface, a coating of hydroxyapatite was obtained after a period of $24 \mathrm{~h}$, which has a thickness of about 2-4 $\mu \mathrm{m}$.
\end{abstract}

Keywords

Hydroxyapatite; Titanium; Biomimetic; Coating.

\section{INTRODUCCIÓN}

En la actualidad, el titanio y sus aleaciones se utilizan de forma extensiva en la fabricación de dispositivos biomédicos implantables que trabajan sometidos a la acción de elevadas cargas biomecánicas. Sin embargo, debido a que existen diferencias significativas entre la composición química y de fases presentada por estos materiales y el tejido óseo, la inserción de estos en el esqueleto humano puede redundar en la ausencia de una unión fuerte entre el hueso y el implante, reacciones a cuerpo extraño y corrosión de la superficie del dispositivo implantado, entre otros ${ }^{[1-3]}$.
Es conocido que ciertas cerámicas bioactivas, entre las que se encuentra la hidroxiapatita (HA), tienen la capacidad de unirse de forma fuerte y estable al hueso, comportamiento vinculado a procesos que facilitan la aposición ósea en sus superficies ${ }^{[4}$ y 5$]$.

En aras de combinar las propiedades mecánicas del titanio con la aptitud de unirse al hueso de estas cerámicas bioactivas, se han ensayado varios métodos para recubrir la superficie metálica, entre los cuales se encuentra el método biomimético ${ }^{[6-9]}$. Este método fue desarrollado a finales de los años 80 y principios de los años 90 del pasado siglo por investigadores japoneses dirigidos por T. Kokubo. El mismo, consiste en la inmersión de superficies bioactivas en un fluido

\footnotetext{
(•) Trabajo recibido el día 10 de Febrero de 2010 y aceptado en su forma final del día 28 de Septiembre 2010.

* Dirección de Química, Centro Nacional de Investigaciones Científicas, Av. 25 y Calle 158, Playa, Apartado Postal 6414, Ciudad de La Habana, Cuba. E-mail: adrian.paz@cnic.edu.cu.

** Instituto Nacional de Tecnología Industrial, Av. General Paz 5445, Apartado Postal B1650WAB, San Martín, Buenos Aires, Argentina.
} 
biológico simulado (SBF por sus siglas en inglés) durante un período de tiempo que permita la formación de una capa apatítica en su superficie. El SBF se caracteriza por poseer una concentración iónica semejante al com-

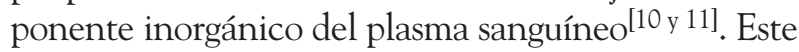
método resulta muy factible debido a que permite obtener recubrimientos homogéneos en toda la superficie en contacto con la disolución, además de resultar tec-

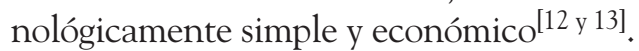

No obstante, la variante del método biomimético desarrollada por Kokubo y colaboradores posee el inconveniente de requerir períodos de tratamiento prolongados, siendo necesario en algunos casos utilizar exposiciones de hasta dos meses ${ }^{[10}$ y 11]. Por ello se han desarrollado numerosos trabajos investigativos destinados a reducir dichos tiempos de tratamiento. Dentro de las variantes más utilizadas para intensificar los procesos se encuentran el incremento de la concentración de iones calcio y fosfato en las disoluciones (conocidas como SCS) y su combinación con tratamientos de activación de las superficies a recubrir ${ }^{[14-16]}$.

A inicios de la presente década, la activación superficial del titanio mediante un tratamiento en una mezcla de peróxido de hidrógeno y ácido clorhídrico le permitió a Wang y colaboradores disminuir el tiempo de deposición de los recubrimientos biomiméticos hasta 7 días ${ }^{[14]}$. Recientemente, Baker y colaboradores lograron disminuir el tiempo de tratamiento a tres días combinando el uso de una disolución sobresaturada en calcio (SCS) con un tratamiento de precalcificación del titanio ${ }^{[15]}$.

En el presente trabajo se modifica la variante del método biomimético desarrollada por Li y colaboradores $^{[16]}$, al incrementarse los valores del $\mathrm{pH}$ inicial de la disolución. El uso de dicha disolución y de un tratamiento de activación que permite obtener una superficie oxidada con elevada bioactividad, posibilita minimizar los tiempos de formación de los recubrimientos y a su vez depositar capas con una elevada cristalinidad y densidad.

\section{PARTE EXPERIMENTAL}

\subsection{Tratamientos realizados al titanio}

Las muestras empleadas como sustratos en la obtención del recubrimiento apatítico se elaboraron a partir de láminas de titanio comercialmente puro $(\mathrm{Ti}$ cp) de $10 \times 4 \times 1 \mathrm{~mm}$. Las probetas fueron pulidas empleando lijas de carburo de silicio $(\mathrm{SiC})$ partiendo de un tamaño de malla de 120 mesh hasta llegar a 400 mesh con el objetivo homogenizar la superficie.

Las muestras pulidas se sometieron a un proceso de limpieza química consistente en su inmersión en una mezcla de $\mathrm{HF} 2,75 \mathrm{~mol} / \mathrm{l}$ y $\mathrm{HNO}_{3} 3,94 \mathrm{~mol} / \mathrm{l}$, con relación v/v 1:1, durante 2 min a temperatura ambiente. Posteriormente, se extrajeron de la mezcla ácida y se enjuagaron con abundante agua destilada. A continuación se colocaron en un baño ultrasónico, en agua destilada, durante $30 \mathrm{~min}$. Para realizar la activación superficial se siguió la variante de tratamiento propuesta por X. X. Wang ${ }^{[14]}$. Las probetas se trataron en una mezcla oxidante de $\mathrm{H}_{2} \mathrm{O}_{2} 8,8 \mathrm{~mol} / \mathrm{l}$ y $\mathrm{HCl}$ 0,1 mol/l, con relación v/v $1: 1$, a $80^{\circ} \mathrm{C}$ durante $30 \mathrm{~min}$. Al finalizar el tratamiento se enjuagaron con agua destilada. Finalmente, las probetas fueron sometidas a un proceso de tratamiento térmico en una mufla, utilizando un régimen de exposición de $400{ }^{\circ} \mathrm{C}$ durante $1 \mathrm{~h}$.

\subsubsection{Preparación de la disolución}

En la elaboración de la disolución con elevado contenido de iones calcio y fosfato empleada en la obtención del recubrimiento se siguió el procedimiento descrito por Li y colaboradores ${ }^{[16]}$. Durante su preparación se disolvieron sucesivamente $\mathrm{CaCl}_{2}$, $\mathrm{NaH}_{2} \mathrm{PO}_{4}$ y $\mathrm{NaHCO}_{3}$ en $500 \mathrm{ml}$ de agua desionizada. El proceso de disolución se llevó a cabo en un baño termostático con agitación, ajustándose la temperatura a $37^{\circ} \mathrm{C}$ y la agitación a $200 \mathrm{rpm}$. Las masas de las sales, así como la concentración iónica teórica de la disolución se muestran en la tabla I.

\subsubsection{Proceso de obtención del recubrimiento}

Las probetas tratadas superficialmente fueron anudadas con un hilo fino de algodón por uno de sus

Tabla I. Masa de las sales (a) y concentración iónica teórica (b) en $500 \mathrm{ml}$ de disolución SCS

Table I. Mass of salt (a) and theoretical ionic concentration (b) in $500 \mathrm{ml}$ of SCS solution

(a) Masa de sales en $\mathbf{5 0 0 ~} \mathrm{ml}$ de SCS (mg)

\begin{tabular}{ccc}
\hline $\mathrm{CaCl}_{2}$ & $\mathrm{NaH}_{2} \mathrm{PO}_{4}$ & $\mathrm{NaHCO}_{3}$ \\
\hline 555 & 150 & 63 \\
\hline
\end{tabular}

(b) Concentración iónica teórica ( $\mathrm{mmol} / \mathrm{l})$

\begin{tabular}{ccccc}
\hline $\mathrm{Na}^{+}$ & $\mathrm{Ca}^{2+}$ & $\mathrm{Cl}^{-}$ & $\mathrm{PO}_{4}{ }^{3-}$ & $\mathrm{HCO}_{3}^{-}$ \\
\hline 4 & 10 & 20 & 2,5 & 1,5 \\
\hline
\end{tabular}


extremos y sumergidas en $500 \mathrm{ml}$ de la disolución (Fig. 1). Con el objetivo de simular las condiciones fisiológicas del cuerpo humano, previo a la inmersión de las probetas en la disolución, se reguló la temperatura del baño a $37^{\circ} \mathrm{C}$ y la agitación a $80 \mathrm{rpm}$. El proceso se extendió durante $24 \mathrm{~h}$. Concluido el tratamiento se extrajeron las probetas de la disolución, se enjuagaron abundantemente con agua destilada y se colocaron en una desecadora.

\subsubsection{Caracterización}

Los valores de $\mathrm{pH}$ de la disolución fueron medidos antes y durante el proceso de recubrimiento, empleando para ello un pHmetro digital Pracitronic modelo MV870, con una precisión de $\pm 0,01$ unidades. Las mediciones fueron realizadas en un intervalo de $30 \mathrm{~min}$.

La determinación del contenido de calcio presente en las disoluciones se realizó con ayuda de un espectrofotómetro de absorción atómica GBC Avanta $\mathrm{P}$, utilizando una llama de aire-acetileno, mientras que para determinar el contenido de fósforo se empleó un espectrofotómetro UV-Visible Spekol 11. Para ello se adicionó a la muestra molibdato de amonio, formándose el ácido molibdofosfórico. Posteriormente, se añadió una mezcla reductora para formar el complejo Azul de molibdeno. La intensidad de color fue medida espectrofotométricamente a $652 \mathrm{~nm}$.

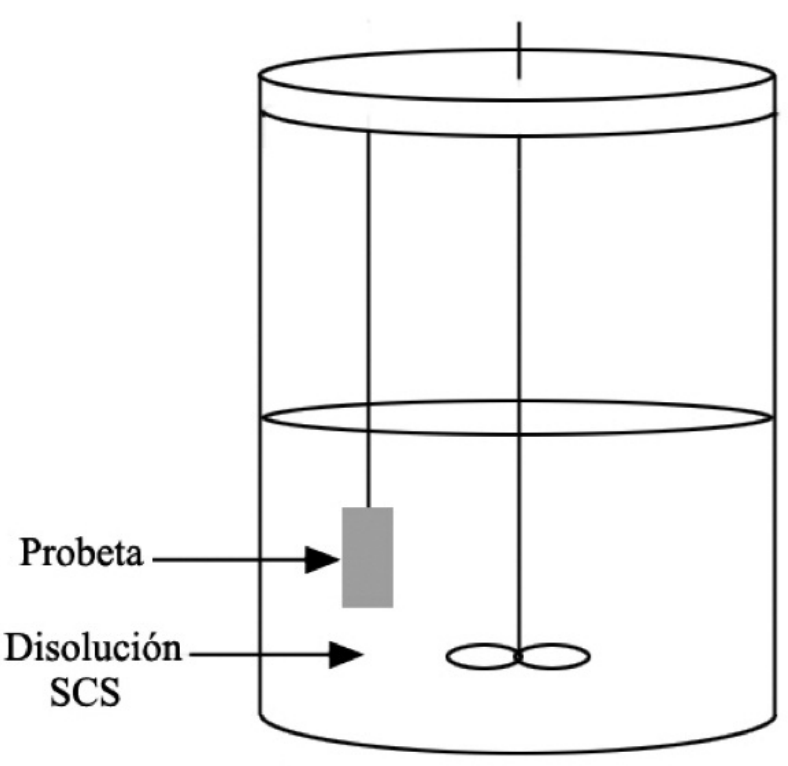

Figura 1. Esquema de inmersión de la probeta en la disolución SCS.

Figure 1. Scheme of the specimen immersion in SCS solution.
En el análisis de los grupos funcionales presentes en el recubrimiento se empleó un Espectrofotómetro infrarrojo por transformada de Fourier marca Nicolet Magna 550 Series II con un accesorio de reflectancia difusa Spectra-Tech. El rango empleado fue de 400 a $4000 \mathrm{~cm}^{-1}$, con una resolución de $4 \mathrm{~cm}^{-1}$.

Las fases presentes tanto en el sustrato como en el recubrimiento se determinaron mediante Difracción de Rayos X (DRX). Para ello se utilizó un equipo marca Thermo Electron modelo XTRA, empleando radiación Ka del cobre. El barrido se llevó a cabo de forma continua con un intervalo angular $2 \theta$ de $10^{\circ}$ a $70^{\circ}$ y un paso de $0,01^{\circ}$. La identificación de las fases presentes se llevó a cabo mediante el método de Hanawalt.

En la realización de las micrografías (MEB) del sustrato y de la superficie del recubrimiento se empleó un microscopio electrónico de barrido marca Tescan TS 5130 SB, con un equipo de Energía Dispersiva de Rayos X (EDX) acoplado. Las muestras fueron recubiertas con una película de $200 \AA$ de oro mediante empolvorizado catódico. La determinación del espesor de los recubrimientos a partir de las micrografías se efectuó con la ayuda del programa Image J v. 1,41 .

\section{RESULTADOS Y DISCUSIÓN}

\subsection{Caracterización del sustrato}

Empleando el método de Hanawalt, se determina que las señales observadas en $2 \theta=35,10^{\circ} ; 38,41^{\circ}$; $40,19^{\circ} ; 53,00^{\circ}$ y $63,02^{\circ}$ corresponden al elemento titanio (Fig. 2) ${ }^{[17]}$.

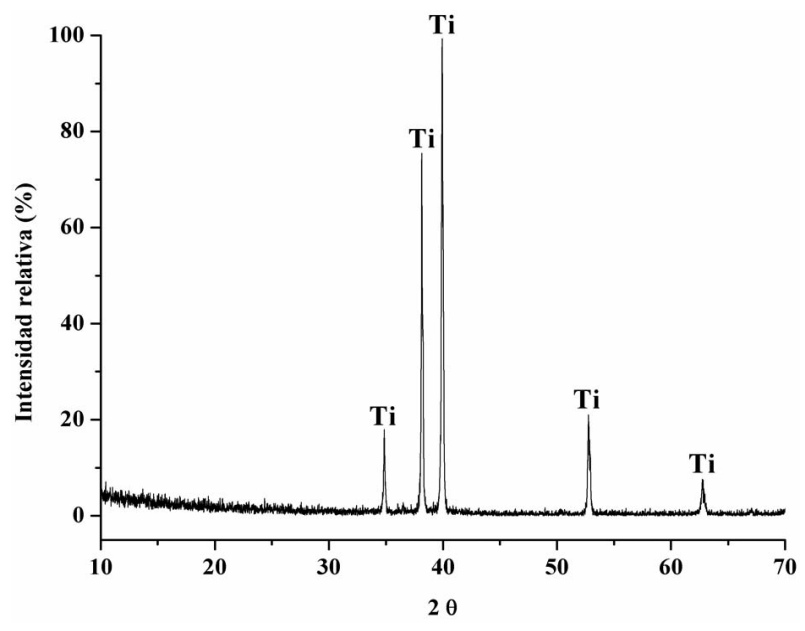

Figura 2. Difractograma del sustrato empleado.

Figure 2. Diffractogram of the substrate used. 
Los ensayos de MEB permitieron caracterizar tanto la superficie pulida como la superficie activada del substrato empleado (Figs. 3 y 4 ).

La micrografía de la superficie pulida (Fig. 3), permite apreciar un relieve formado por crestas y valles alineados en la dirección en que se desplazaron las partículas abrasivas durante el pulido. El análisis de la composición elemental del titanio empleado como substrato fue determinada mediante la técnica de EDX (Tabla II). La ausencia de elementos de aleación (con la excepción de 0,32 \% de hierro), permite clasificarlo como titanio comercialmente puro, lo cual corrobora los resultados obtenidos mediante el ensayo de DRX.

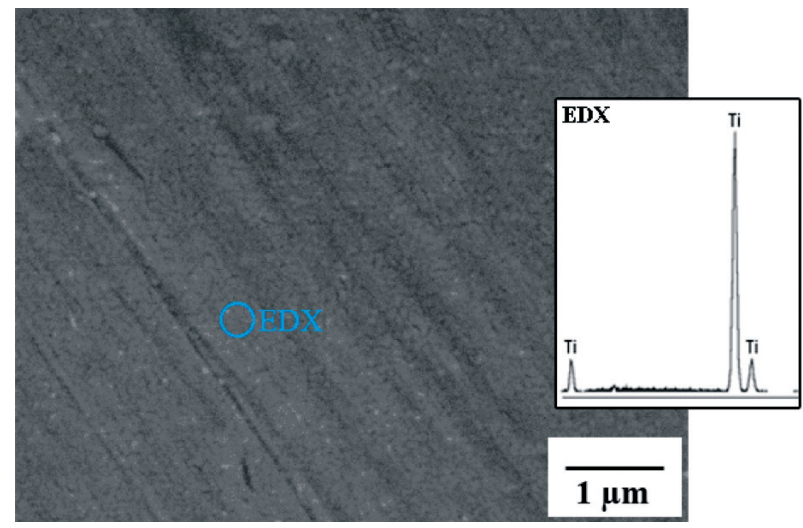

Figura 3. Micrografía de la superficie pulida del sustrato y ensayo de EDX correspondiente.

Figure 3. Micrograph of the polished surface of the substrate and corresponding EDX testing.

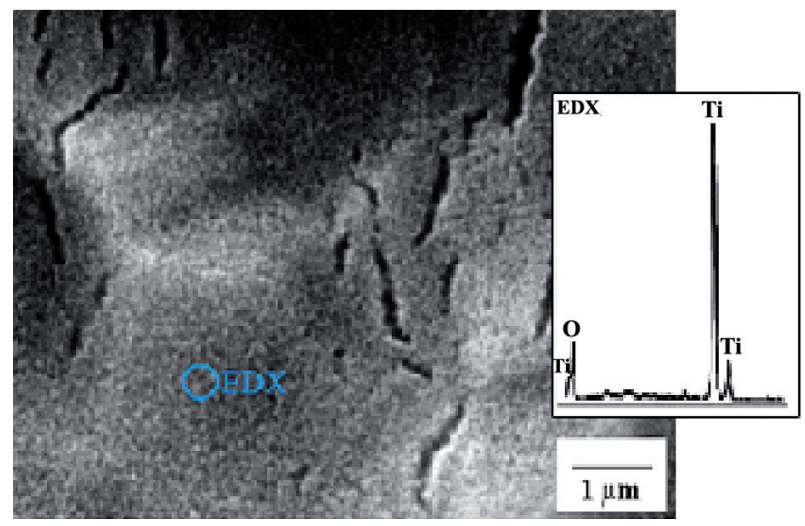

Figura 4. Micrografía de la superficie posterior al tratamiento térmico y ensayo de EDX correspondiente.

Figure 4. Micrograph of the surface after heat treatment and corresponding EDX testing.
Tabla II. Composición (\% Atómico) del titanio utilizado como sustrato

Table II. Composition (Atomic \%) of the titanium used as substrate

\begin{tabular}{ccc}
\hline Elemento & Masa (\% At) & $\begin{array}{c}\text { Masa (\% At) } \\
\text { Error }\end{array}$ \\
\hline $\mathrm{Al}$ & 0,03 & $+/-0,01$ \\
$\mathrm{Si}$ & 0,05 & $+/-0,01$ \\
$\mathrm{~K}$ & 0,08 & $+/-0,01$ \\
$\mathrm{Ca}$ & 0,43 & $+/-0,02$ \\
$\mathrm{Ti}$ & 99,1 & $+/-0,3$ \\
$\mathrm{Fe}$ & 0,32 & $+/-0,04$ \\
Total & 100,00 & \\
\hline
\end{tabular}

La oxidación química con la mezcla de $\mathrm{H}_{2} \mathrm{O}_{2}$ y $\mathrm{HCl}$, combinado con un posterior tratamiento térmico a $400{ }^{\circ} \mathrm{C}$ durante $1 \mathrm{~h}$, posibilita obtener una capa homogénea, presumiblemente de anatasa según lo reportado por varios autores para la oxidación térmica del titanio al aire ${ }^{[14}$ y 18$]$.

La micrografía de la superficie posterior al tratamiento térmico (Fig. 4) revela la ausencia de las crestas y valles formados en el proceso de pulido, observándose además la presencia de microgrietas, generadas presumiblemente durante el proceso de tratamiento térmico, distribuidas en el fondo, paredes y bordes exteriores de los cráteres.

\subsection{Caracterización de la disolución}

Se realizó un estudio del comportamiento del pH desde la disolución de las sales hasta la conclusión del proceso con la sustracción de las muestras del baño. Los valores medios de $\mathrm{pH}$ en función del tiempo se pueden apreciar en la figura 5 .

En las primeras $2 \mathrm{~h}$ de tratamiento se genera un incremento sensible del $\mathrm{pH}$ hasta alcanzar un valor máximo de 6,52, seguido de una disminución brusca de este factor en un período de tiempo corto $(5,85$ a las $4 \frac{1}{2} \mathrm{~h}$ de tratamiento). Posteriormente, se produce una disminución gradual, hasta concluir el tratamiento a las $24 \mathrm{~h}$.

Durante el proceso de deposición, coincidiendo con la disminución brusca del valor de $\mathrm{pH}$ a las $2 \mathrm{~h}$, se observó la aparición de precipitados en forma de pequeñas partículas blancas, tanto en la superficie de las probetas como en el seno de la disolución.

El comportamiento del $\mathrm{pH}$ de la disolución se explica por la disociación de iones inorgánicos y por 


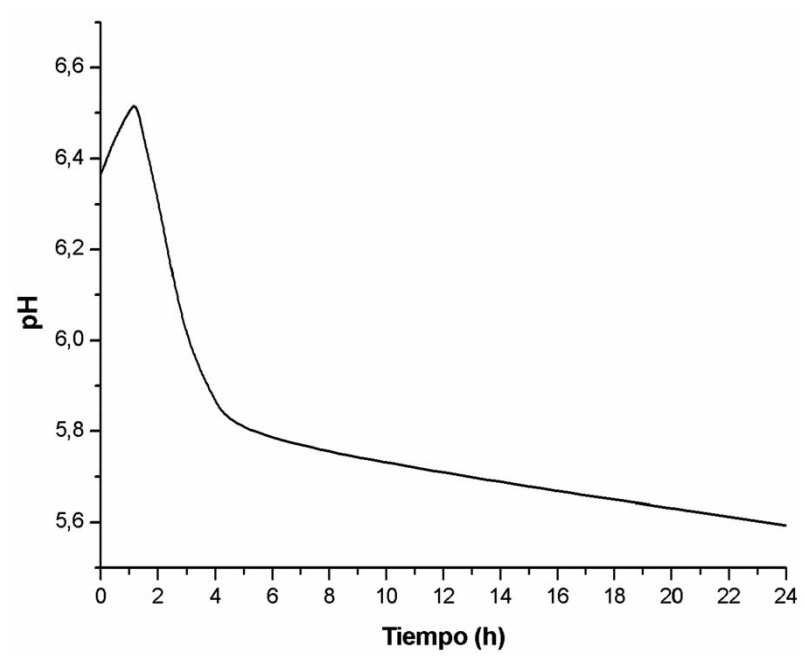

Figura 5. Comportamiento del valor medio del $\mathrm{pH}$ de la disolución SCS.

\section{Figure 5. Behavior of the mean $\mathrm{pH}$ value of SCS solution.}

la posterior formación de núcleos apatíticos. La condición ligeramente ácida de la disolución inicial tiene su origen en la disociación del anión dihidrógenofosfato $\left(\mathrm{H}_{2} \mathrm{PO}_{4}^{-}\right)$, ecuación (1). Esta reacción incorpora iones $\mathrm{H}^{+}$al medio, disminuyendo el valor del $\mathrm{pH}$.

$$
\mathrm{H}_{2} \mathrm{PO}_{4}^{-} \leftrightarrow \mathrm{HPO}_{4}^{2-}+\mathrm{H}^{+}
$$

El incremento del valor de $\mathrm{pH}$ en la etapa inicial de la inmersión de las muestras está justificado por la reacción de los iones $\mathrm{HCO}_{3}{ }^{-}$generados durante la disociación del $\mathrm{NaHCO}_{3}$ con los iones $\mathrm{H}^{+}$, ecuación (2), provocando el consumo de estos últimos.

$$
\mathrm{H}^{+}+\mathrm{HCO}_{3}^{-} \leftrightarrow \mathrm{H}_{2} \mathrm{O}+\mathrm{CO}_{2}
$$

Luego de alcanzarse un valor máximo de $\mathrm{pH}$, se observó una brusca disminución de los valores de dicho parámetro. Este fenómeno debe encontrarse relacionado con la formación del precipitado de hidroxiapatita, ecuación (3), lo cual provoca un rápido consumo de iones $\mathrm{OH}^{-}$presentes en la disolución, pasando a formar parte del precipitado y del recubrimiento, a la vez que incorpora iones $\mathrm{H}^{+}$al medio $^{[16]}$.

$$
10 \mathrm{Ca}^{2+}+6 \mathrm{H}_{2} \mathrm{PO}_{4}^{-}+2 \mathrm{H}_{2} \mathrm{O} \leftrightarrow \mathrm{Ca}_{10}\left(\mathrm{PO}_{4}\right)_{6}(\mathrm{OH})_{2}+14 \mathrm{H}^{+}
$$

Mediante la aplicación de técnicas espectrofotométricas, se lleva a cabo la determinación de las concentraciones iniciales y finales de los elementos calcio y fósforo presentes en la disolución, cuyos valores se pueden apreciar en la figura 6 .

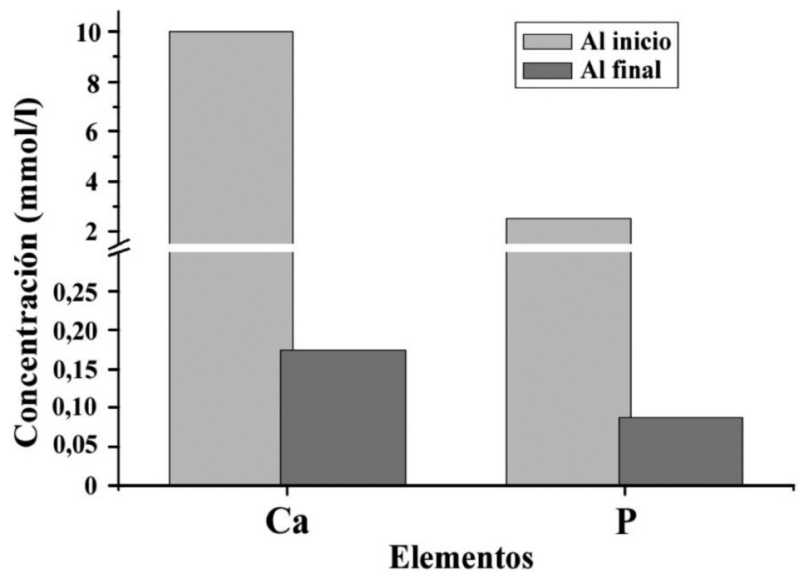

Figura 6. Variación de las concentraciones promedios inicial y final de calcio y fósforo en la disolución.

Figure 6. Variation of the average initial and final concentrations of calcium and phosphorus in the solution.

La disolución de partida contiene a los mencionados elementos en una concentración de $10 \mathrm{mmol} / \mathrm{l}$ y $2,5 \mathrm{mmol} / \mathrm{l}$ respectivamente. Luego de culminado el proceso, se observa una marcada disminución en las concentraciones de estos elementos, obteniéndose como promedio $1,75 \cdot 10^{-1} \mathrm{mmol} / \mathrm{l}$ para el calcio y $8,74 \cdot 10^{-2} \mathrm{mmol} / \mathrm{l}$ para el fósforo (Fig. 6).

La apreciable disminución que sufre la concentración de iones $\mathrm{Ca}^{2+}$ y $\mathrm{PO}_{4}^{3-}$ se debe a la formación de precipitados de fosfatos de calcio en la disolución y al crecimiento de un recubrimiento apatítico en la superficie de la muestras de titanio.

\subsection{Caracterización del recubrimiento}

El espectro infrarrojo obtenido del recubrimiento muestra las bandas fundamentales correspondientes a los grupos principales de la hidroxiapatita (Fig. 7).

Entre la región de los $1.040 \mathrm{~cm}^{-1}$ y $1.091 \mathrm{~cm}^{-1} \mathrm{se}$ encuentra la señal más importante de la hidroxiapatita, la vibración antisimétrica de los fosfatos de calcio $\cup_{3}{ }^{\text {as }}\left(\mathrm{PO}_{4}{ }^{3-}\right)$, mientras que la vibración simétrica aparece en los $952 \mathrm{~cm}^{-1}$. Sobre los 561 y $606 \mathrm{~cm}^{-1}$ se encuentra la vibración antisimétrica $\cup_{4}^{\text {as }}\left(\mathrm{PO}_{4}^{3-}\right.$ ). Se observa además una banda sobre los $635 \mathrm{~cm}^{-1}$, la cual corresponde al doblaje fuera del plano $(\rho)$ del grupo $\mathrm{OH}^{-}$estructural. La señal situada sobre los 1643 $\mathrm{cm}^{-1}$ pertenece a agua de humedad ${ }^{[19}$ y 20$]$. Es necesario destacar, que la estructura fina e intensa de las señales pertenecientes al grupo fosfato representan 


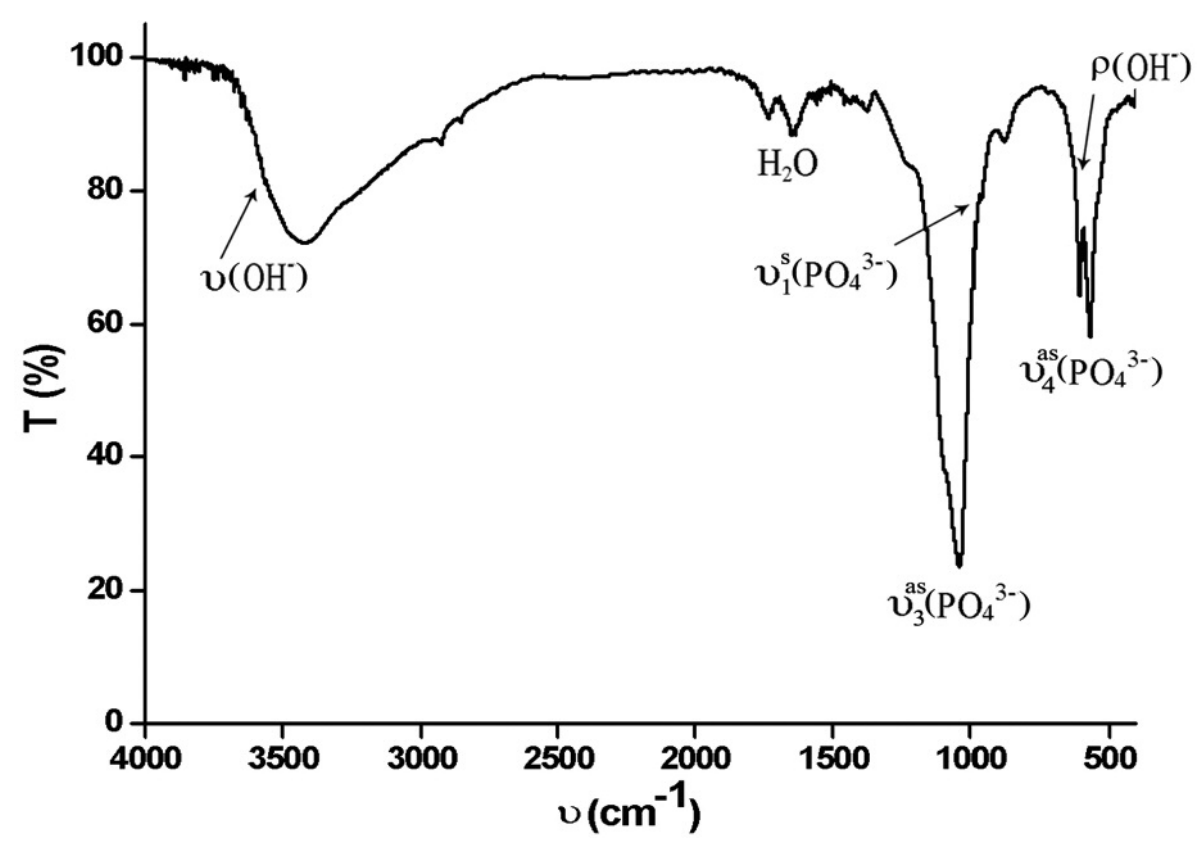

Figura 7. Espectro infrarrojo del recubrimiento.

Figure 7. Infrared spectrum of the coating.

un claro indicio de la elevada cristalinidad que posee el recubrimiento obtenido ${ }^{[21]}$.

El difractograma de la superficie de titanio tratado con la disolución SCS permite detectar la presencia de una nueva fase cristalina correspondiente a la hidroxiapatita (Fig. 8). Las señales características de la hidroxiapatita se aprecian en $2 \theta=25,91^{\circ} ; 32,18^{\circ} \mathrm{y}$ $49,52^{\circ}$. Además, se observan los picos correspondientes al titanio del sustrato ${ }^{[17]}$. La elevada intensidad de la señal ubicada en $2 \theta=25,91^{\circ}$, perteneciente

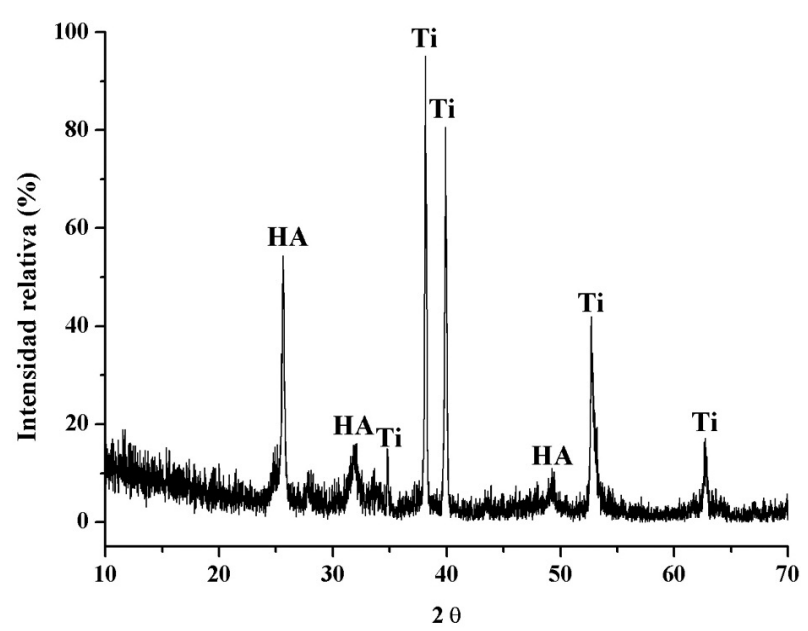

Figura 8. Difractograma del recubrimiento.

Figure 8. Diffractogram of the coating. al plano (002), es provocada por la orientación preferencial del eje cristalográfico $c$ de los cristales de HA, debido al crecimiento perpendicular sobre la superficie del sustrato. Además, la estructura fina de dicha señal permite corroborar las características cristalinas que presenta el recubrimiento formado.

Las micrografías de la superficie de las muestras sometidas al proceso de recubrimiento (Fig. 9) muestran la formación de depósitos densos, con la aparición de algunas aglomeraciones de partículas cristalinas (zonas claras). También se observan algunos poros y grietas característicos de los recubrimientos depositados mediante el método biomimético ${ }^{[21]}$.

El análisis de la composición elemental del recubrimiento depositado en la superficie del titanio permite determinar la presencia de calcio, fósforo y oxígeno en cantidades apreciables, además del titanio utilizado como sustrato. La presencia de los mencionados elementos confirma la existencia de un fosfato de calcio (hidroxiapatita) en la superficie del material. Este resultado coincide con la caracterización realizada mediante las técnicas de DRX y FT-IR.

La micrografía de la sección transversal de las probetas corrobora la formación del recubrimiento en la superficie del sustrato de titanio (Fig. 10) con un espesor entre $2-4 \mu \mathrm{m}$. Además, el recubrimiento se encuentra constituido, en dicha zona, por partículas nanométricas y submicrométricas con dimensiones inferiores a $200 \mathrm{~nm}$ (Fig. 10). A continuación del recubrimiento apatítico se aprecia una capa delgada 

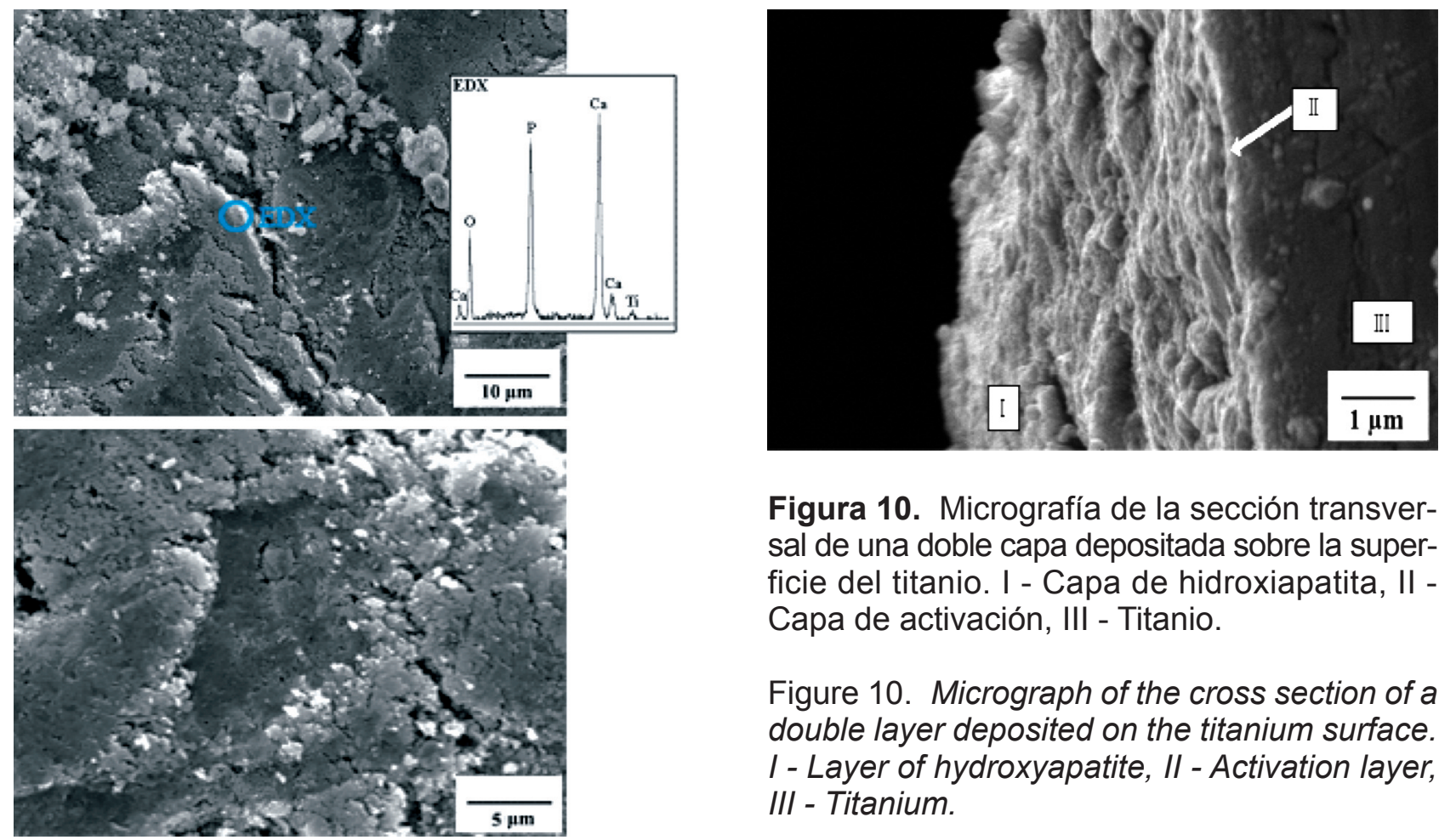

Figura 10. Micrografía de la sección transversal de una doble capa depositada sobre la superficie del titanio. I - Capa de hidroxiapatita, II Capa de activación, III - Titanio.

Figure 10. Micrograph of the cross section of a double layer deposited on the titanium surface. I - Layer of hydroxyapatite, II - Activation layer, III - Titanium.

Figura 9. Micrografías de la superficie del recubrimiento y ensayo de EDX correspondiente.

Figure 9. Micrographs of the coated surface and corresponding EDX testing.

de color blanco, caracterizada por su homogeneidad (zona II de la figura 10) y por último el titanio que sirve como sustrato, en el cual se pueden observar la presencia de microgrietas. Este fenómeno puede estar relacionado con las tensiones generadas por el régimen de calentamiento empleado (zona III de la figura 10).

\section{CONCLUSIONES}

- Se obtuvo un recubrimiento apatítico sobre la superficie del titanio activada mediante la combinación de un tratamiento químico con un posterior tratamiento térmico, a partir de una variante del método biomimético que utiliza una disolución con elevado contenido de calcio y fósforo.

- Los ensayos de FT-IR y DRX, permitieron determinar que la fase fundamental en el recubrimiento obtenido es hidroxiapatita con elevada cristalinidad (fase similar a la del componente inorgánico del tejido óseo). Además, el depósito se distingue por poseer un espesor entre $2-4 \mu \mathrm{m}$, resultar denso y encontrarse

constituido fundamentalmente por los elementos calcio, fósforo y oxígeno.

- Las características antes enunciadas permiten prever que el recubrimiento desarrollado debe poseer un buen comportamiento in vivo, debido a que debe activar el proceso de aposición ósea y actuar como barrera, evitando la liberación de iones del substrato al medio circundante.

\section{Agradecimientos}

Los autores quieren agradecer la colaboración recibida por parte de INTI-Mecánica e INTI-Procesos Superficiales, al MINCYT-CITMA (Proyecto CU/08/13) y a la Cancillería Argentina por el financiamiento recibido (FO-AR 5714).

\section{REFERENCIAS}

[1] N.G Boon Sing, I. Annergren, A.M. Soutar, K.A. Khor y A. Jarfors, Biomaterials 26 (2005) 1.087-1.095.

[2] H. Güleryüz y H. Cimenoglu, Biomaterials 25 (2004) 3.325-3.333.

[3] D.M. Liu, Q. Yang y T. Troczynski, Biomaterials 23 (2002) 3.103-3.111.

[4] L.L. Hench y R.J. Splinter, J. Biomed. Mater. Res. 2 (1972) 1.17-1.41. 
[5] K. Søballe, S. Overgaard y E.S. Hansen, J. LongTerm Eff. Med. Implt. 9 (1999) 131-151.

[6] S.W.K. Kweh, K.A. Khor y P. Cheang, Biomaterials 21 (2000) 1.223-1.234.

[7] C.Y. Yang, R.M. Lin, B.C. Wang, T.M. Lee, E. Chang, Y.S. Hang y P.Q. Chen, J. Biomed. Mater. Res. 37 (1997) 335-345.

[8] B. Story y A. Burgess, US Patent 5730598, Prosthetic implants coated with hydroxyapatite and process for treating prosthetic implants plasma-sprayed with hydroxyapatite (1998).

[9] W. Song, Y.K. Jun, Y. Han y S.H. Hong, Biomaterials 25 (2004) 3.341-3.349.

[10] T. Kokubo, C. Ohtsuki, S. Kotani, T. Kitsugi y T. Yamamuro, Proc. Bioceramics, vol. 2, Heidelberg, Alemania, G. Heimke (Ed.), German Ceramics Society, Cologne, Alemania, 1990, pp. 113-120.

[11] T. Kokubo. H. Kushitani, S. Sakka, T. Kitsugi y T. Yamamuro, J. Biomed. Mater. Res. 24 (1990) 721-734.

[12] B. Feng. J.Y. Chen, S.K.L. Qic, J. He, Z. Zhao y X. Zhang, Biomaterials 23 (2002) 173-179.
[13] M. Kawashita, M. Nakao, M. Minoda, H.M. Kim, T. Beppu y T. Miyamoto, Biomaterials 24 (2003) 77-84.

[14] X.X. Wang, S. Hayakawa, K. Tsuru y A. Osaka, J. Biomed. Mater. Res 54 (2001) 172-178.

[15] K.C. Baker, M.A. Anderson, S.A. Oehlke, A.I. Astashkina, D.C. Haikio, J. Drelichb y S.W. Donahue, Mat. Sci. Eng. C. 26 (2006) 1.351-1.360.

[16] F. Li, Q.L. Feng, F.Z. Cui, H.D. Li y H. Schubert, Surf. Coat. Tech. 154 (2002) 88-93.

[17] International Centre for Diffraction Data, PCPDFWin, v. 1.30, JCPDS, No. 44-1294 y 74-0565. (1998).

[18] J. Alcisto, A. Enriquez, H. García, S. Hinkson, M. Hahn, J. Foyos, J. Ogren y E.E. Lee, Eng. Fail. Anal. 11 (2004) 881-916.

[19] E. Peón. G. Fuentes, J.A. Delgado, L. Morejón, A. Almirall y R. García, Latin American Applied Research 34 (2004) 225-228.

[20] B.O. Fowler, Inorg. Chem. 13 (1974) 194-207.

[21] F. Barrere, P. Layrolle, C.A. van Blitterswijk y K. de Groot, Biomaterials 23 (2002) 2.211-2.220. 